\title{
First-Line Anlotinib Combined with Stereotactic Body Radiation Therapy for an Octogenarian with Pulmonary Carcinosarcoma
}

\author{
Long Liang ${ }^{1, *}$ \\ Taiyu Chen ${ }^{2, *}$ \\ Tao Ren ${ }^{3}$ \\ Mian $\mathrm{Mao}^{4}$ \\ Keli Wang ${ }^{5}$ \\ Chunhan Tang ${ }^{2}$ \\ Juan Xiao' \\ Hongyuan Jia' \\ Wenli Chen' \\ Qifeng Wang $\mathbb{D}^{\prime}$ \\ Tao $\mathrm{Li}^{\prime}$
}

'Department of Radiation Oncology, Sichuan Cancer Hospital and Institution, Sichuan Cancer Center, School of Medicine, University of Electronic Science and Technology of China, Radiation Oncology Key Laboratory of Sichuan Province, Chengdu, People's Republic of China; ${ }^{2}$ Clinical Medical College of Chengdu Medical College, Chengdu, 610500, People's Republic of China; ${ }^{3}$ Oncology Department, Clinical Medical College and The First Affiliated Hospital of Chengdu Medical College, Chengdu, 610500, People's Republic of China; ${ }^{4}$ Department of Pharmacy, Sichuan Cancer Hospital \& Institute, Sichuan Cancer Center, School of Medicine, University of Electronic Science and Technology of China, Chengdu, People's Republic of China; ${ }^{5}$ Clinical Medical College of Southwest Medical University, Luzhou, 646000, People's Republic of China

*These authors contributed equally to this work

Correspondence: Tao $\mathrm{Li}$ $\mathrm{Tel} / \mathrm{Fax}+862885420173$

Email litaoxmf@I26.com

\begin{abstract}
Pulmonary carcinosarcoma (PC) is a rare and highly malignant type of non-small cell lung cancer (NSCLC) that is insensitive to radiotherapy and chemotherapy and has a poor prognosis. Here, we report a case of an 88-year-old patient with inoperable PC and a history of cerebral infarction who was treated with first-line anlotinib combined with stereotactic body radiation therapy (SBRT). The therapeutic response has sustained for 10 months. Our work suggests that SBRT combined with anlotinib may be a safe and effective treatment strategy for octogenarians with PC.
\end{abstract}

Keywords: pulmonary carcinosarcoma, SBRT, anlotini

\section{Introduction}

The World Health Organization classifies pulmonary carcinosarcoma (PC) as a subtype of sarcomatoid carcinoma with poor cell differentiation. It partially contains or completely lacks conventional components of non-small cell lung cancer (NSCLC), ${ }^{1}$ PC is very rare and highly invasive in clinical practice, also has a poor prognosis. Identification of effective and safe treatments for patients with advanced $\mathrm{PC}$ is an urgent clinical problem. We report an octogenarian patient with inoperable PC and a history of cerebral infarction who achieved a partial response (PR) after receiving local stereotactic body radiation therapy (SBRT) combined with first-line anlotinib.

\section{Case Report}

An 88-year-old male patient with a 60 pack-year history of smoking suffered cerebral infarction in August 2020. Computed tomography (CT) scans showed an $11.0 \mathrm{~cm} \times 5.6 \mathrm{~cm}$ left upper lobe lesion, and necrosis was visible in the pleura (Figure 1). Histological examination showed squamous cell carcinoma and osteosarcoma, suggesting carcinosarcoma. Immunohistochemistry (IHC) showed heteroepithelial nests that were positive for P40, PCK, CK5/6, SATB2, SMA (partial), and negative for TTF-1, CK7, Dcsmin, CD34, STAT6, ALK-V, and ROS-1. Our gene detection method showed that EGFR, ALK, ERBB2, BRAF, MET, ROS1, RET, and $K R A S$ were all wild-type and unamplified. So the diagnosis was left upper lobe pulmonary carcinosarcoma (cT4N0M0 stage II AJCC 8th).

The patient was deemed inoperable after being reviewed by the Institutional MDT group. In October 2020, he was treated with $10 \mathrm{mg}$ anlotinib per day orally, 


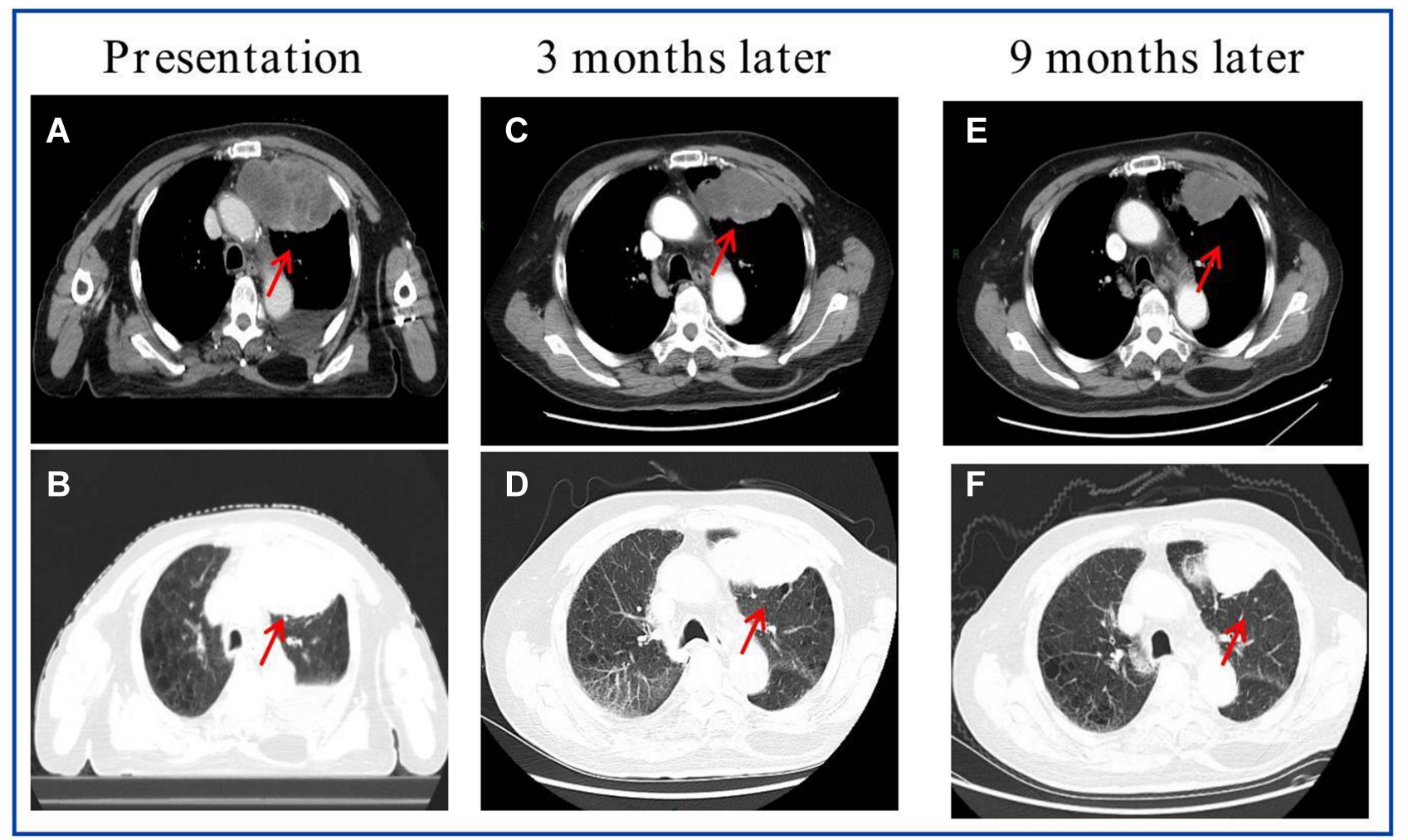

Figure I Chest CT scans demonstrating a left upper lung lobe lesion, (A and B) at diagnosis, (C and D) following 3 months of anlotinib treatment, and (E and $\mathbf{F})$ following 9 months of anlotinib treatment.

combined with SBRT at the same time. The maximum intensity projection of gross lesion was defined as gross tumor volume (GTV). The planning target volume (PTV) was determined based on the GTV2 with a margin of $5 \mathrm{~mm}$, GTV1 has no PTV.Treatment was delivered using volumetric modulated arc therapy (VMAT). Simultaneously integrated boost radiotherapy(SIB) was used on the target area (Figure 2), which approach to the hypoxic center of a tumor may have benefits for treating resistant clones while also allowing for lower radiation doses that are safe for normal tissues. ${ }^{2}$ GTV1 $=65.5 \mathrm{~Gy} /$ $10 \mathrm{~F}$ [biologic equivalent dose (BED) $=109.4 \mathrm{~Gy}$ ]. GTV2 $=50 \mathrm{~Gy} / 10 \mathrm{~F}$ (BED=75 Gy). The SBRT plan was optimized to achieve a D95 PTV (dose received by $95 \%$ volume) of $100 \%$. The mean bilateral lung dose was 8.3 Gy. The portion of lung receiving a dose of 5 Gy (V5) was $46.8 \%, 10$ Gy (V10) was 35.3\%, and 20 Gy (V20) was $11.8 \%$. The maximum dose to the spinal cord was $6.9 \mathrm{~Gy}$. The patient tolerated the therapy well without grade $2-5$ toxicities throughout the treatment period. The lesion was

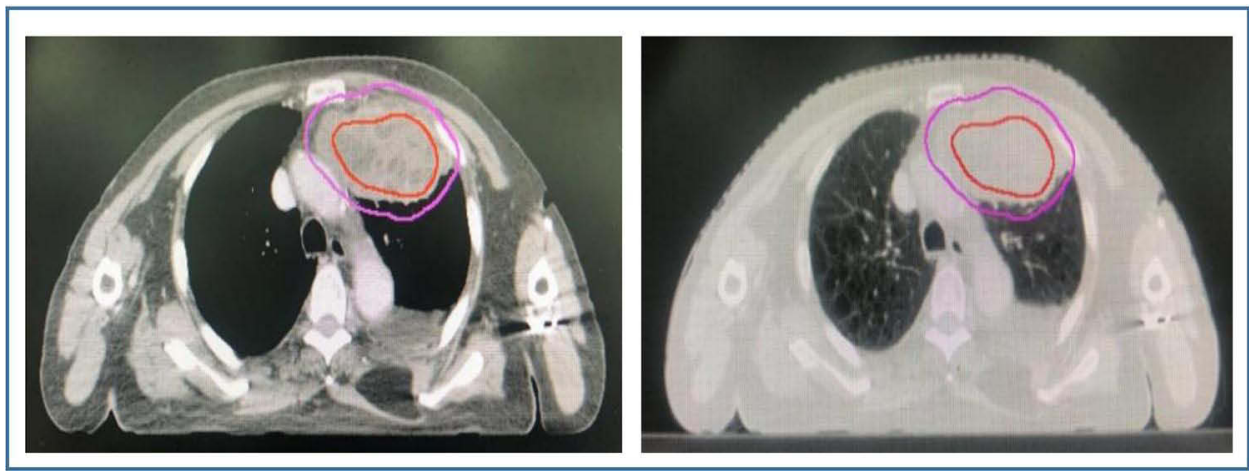

Figure 2 Treatment planning: image of GTV for the SIB technique; the internal area is GTVI with dose at the level of 65.5 Gy (red), and the peripheral area is GTV2 shown at the level of $50 \mathrm{~Gy}$ (pink). 
decreased on chest CT scans 3 and 9 months later, and the response evaluation was PR (Figure 1). Anlotinib treatment was ongoing, and during this period the patient presented with Common Terminology Criteria for Adverse Events grade 2 elevated blood pressure, constipation, and other discomfort. Imaging showed PR with no grade $\geq 3$ adverse effects.

\section{Discussion}

Pulmonary carcinosarcoma is composed of both NSCLC (especially squamous cell carcinoma or adenocarcinoma) and heterogeneous sarcoma components (such as rhabdomyosarcoma and chondrosarcoma). It accounts for less than $1 \%$ of malignant thoracic tumors. ${ }^{3}$ In one study using the SEER database, 411 patients with PC were analyzed from 1973 to 2012. The median age was 67 years, and the overall survival (OS) for patients with localized disease compared to patients with non-localized disease was 31 months vs 6 months, respectively, ${ }^{4}$ suggesting that the prognosis of patients with advanced carcinosarcoma is extremely poor. For patients with advanced or inoperable pulmonary carcinosarcomas, the standard treatment strategy has not yet been well defined, ${ }^{3}$ especially for elderly patients.

Due to the rarity of $\mathrm{PC}$, there have been no large-scale randomized clinical trials for genetic testing and targeted therapy. However, several individual reports using antitumor angiogenesis treatment have shown some efficacy. Tanimoto et al reported a metastatic case treated with pazopanib with 6 months of progression-free survival (PFS). ${ }^{5}$ Furthermore, another case report used apatinib combined with endostatin in PC, and the PFS was up to 6 months. ${ }^{6}$ At present, anti-tumor angiogenesis therapy for lung sarcoma is mainly used as a second-line treatment or beyond. It shows clinical benefits, but PFS is limited. In this case, the patient was treated with first-line anlotinib in combination with SBRT, and the PFS was longer than that reported for anti-tumor angiogenesis therapy. Anlotinib is a small-molecule, multi-target tyrosine kinase inhibitor for tumor angiogenesis and growth. The ALTER0303 study reported that third-line anlotinib for advanced refractory NSCLC resulted in significantly longer OS (9.6 months vs 6.3 months) and PFS (5.4 months vs 1.4 months) compared with the placebo. ${ }^{7}$

The patient reported here could not be treated surgically due to age and a history of cerebral infarction. Over the past decade, SBRT has become an effective treatment for inoperable lung cancer. It has been demonstrated to provide survival benefits superior to conventional fractionated radiotherapy, especially for elderly oligometastatic patients. $^{8-10}$ There are some reports demonstrates that SBRT might be safe and effective for soft tissue sarcoma. ${ }^{11-13}$ Huang et al reported a case of an octogenarian with PC treated with SBRT, at a dose of $60 \mathrm{~Gy}$ in eight fractions (7.5 Gy per fraction). The treatment was evaluated as PR and lasted for 2 years. ${ }^{14}$ Therefore, SBRT is an effective local treatment for medically inoperable PC patients.

The most common adverse effects of anlotinib include hypertension, hand-foot syndrome (HFSR), fatigue, diarrhea, and anorexia. ${ }^{15}$ In this case, there were no adverse events that required discontinuation or dose reduction, indicating that anlotinib is safe for elderly patients.

In summary, carcinosarcoma (including squamous cell carcinoma and osteosarcoma components) is a rare subtype of NSCLC, and there are difficulties and limitations in diagnosis and treatment. In the current case, despite advanced age and cerebral infarction, the use of SBRT combined with oral anlotinib was effective and safe. PFS has sustained for 10 months, and the treatment did not cause serious adverse reactions. Large-scale clinical studies are required to confirm our findings.

\section{Ethical Approval}

Institutional approval was not required to publish the case details. The patient has provided written informed consent for the case details to be published.

\section{Acknowledgment}

We are grateful to the patient and her family. This work was funded by China International Medical Foundation (2019-N-11-25).

\section{Disclosure}

The authors have nothing to disclose.

\section{References}

1. Baldovini C, Rossi G, Ciarrocchi A. Approaches to tumor classification in pulmonary sarcomatoid carcinoma. Lung Cancer. 2019;10:131-149. doi:10.2147/LCTT.S186779

2. Crane $\mathrm{CH}$. Hypofractionated ablative radiotherapy for locally advanced pancreatic cancer. $J$ Radiat Res. 2016;57:i53-i57. doi:10.1093/jrr/rrw016

3. Travis WD, Brambilla E, Nicholson AG, et al. The 2015 World Health Organization classification of lung tumors: impact of genetic, clinical and radiologic advances since the 2004 classification. J Thorac Oncol. 2015;10(9):1243-1260. doi:10.1097/JTO.0000000000000630 
4. Ersek JL, Symanowski JT, Han Y, et al. Pulmonary carcinosarcoma: a surveillance, epidemiology, and end results (SEER) analysis. Clin Lung Cancer. 2020;21(2):160-170. doi:10.1016/j.cllc.2019.07.001

5. Tanimoto A, Takeuchi S, Kotani H, et al. Pulmonary carcinosarcoma showing an obvious response to pazopanib: a case report. BMC Pulm Med. 2018;18(1):193. doi:10.1186/s12890-018-0757-7

6. Tang H, Wu Y. PUB008 successful treatment with apatinib plus human endostatin for refractory recurrent advanced pulmonary carcinosarcoma: a case report. J Thorac Oncol. 2017;12(11):S2367. doi:10.1016/j.jtho.2017.09.1871

7. Han B, Li K, Wang Q, et al. Effect of anlotinib as a third-line or further treatment on overall survival of patients with advanced non-small cell lung cancer: the ALTER 0303 phase 3 randomized clinical trial. JAMA Oncol. 2018;4(11):1569-1575. doi:10.1001/ jamaoncol.2018.3039

8. Newman NB, Sherry AD, Byrne DW, et al. Stereotactic body radiotherapy versus conventional radiotherapy for early-stage small cell lung cancer. J Radiat Oncol. 2019;8(2):239-248. doi:10.1007/ s13566-019-00395-x

9. Kreinbrink P, Blumenfeld P, Tolekidis G, et al. Lung stereotactic body radiation therapy (SBRT) for early-stage nonsmall cell lung cancer in the very elderly ( $\geq 80$ years old): extremely safe and effective. $J$ Geriatr Oncol. 2017;8(5):351-355. doi:10.1016/j. jgo.2017.07.002
10. Cuccia F, Mazzola R, Pastorello E, et al. SBRT for elderly oligometastatic patients as a feasible, safe and effective treatment opportunity. Clin Exp Metastasis. 2021;38(5):475-481. doi:10.1007/s10585-02110122-x

11. Parsai S, Lawrenz J, Kilpatrick S, et al. Early outcomes of preoperative 5-fraction radiation therapy for soft tissue sarcoma followed by immediate surgical resection. Adv Radiat Oncol. 2020;5:1274-1279. doi:10.1016/j.adro.2020.06.024

12. Allen M, Silvino D, Kamrava M, et al. Hypofractionated radiation therapy and wound healing after massive sarcoma resection: case report and review of the literature. Int $J$ Surg Case Rep. 2021;83:106005. doi:10.1016/j.ijscr.2021.106005

13. Mizumatsu S, Ryu H, Nomura K, et al. Treating low-grade myxofibrosarcoma with stereotactic body radiation therapy using CyberKnife. Cureus. 2021;13(7):e16393. doi:10.7759/cureus.16393

14. Huang W-L, Huang Y-L, Kao C-N, et al. Stereotactic body radiation therapy for an octogenarian with pulmonary carcinosarcoma. Thorac Cancer. 2021;12(9):1445-1448. doi:10.1111/1759-7714.13922

15. Gao Y, Liu P, Shi R. Anlotinib as a molecular targeted therapy for tumors. Oncol Lett. 2020;20(2):1001-1014. doi:10.3892/ ol.2020.11685

\section{Publish your work in this journal}

Cancer Management and Research is an international, peer-reviewed open access journal focusing on cancer research and the optimal use of preventative and integrated treatment interventions to achieve improved outcomes, enhanced survival and quality of life for the cancer patient.
The manuscript management system is completely online and includes a very quick and fair peer-review system, which is all easy to use. Visit http://www.dovepress.com/testimonials.php to read real quotes from published authors. 